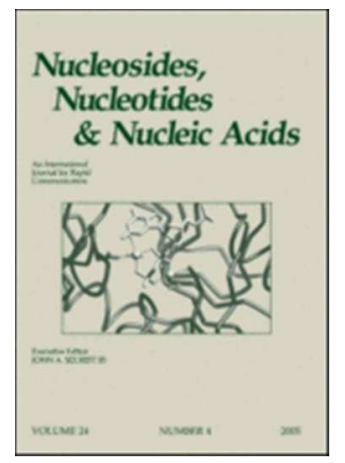

\title{
Discovery profiling and bioinformatics analysis of serum microRNA in Mitochondrial NeuroGastroIntestinal Encephalomyopathy (MNGIE)
}

\begin{tabular}{|r|l|}
\hline Journal: & Nucleosides, Nucleotides and Nucleic Acids \\
\hline Manuscript ID & LNCN-2018-0014.R1 \\
\hline Manuscript Type: & Conference Proceedings \\
\hline Date Submitted by the Author: & 08-Jun-2018 \\
\hline Keywords: List of Authors: & $\begin{array}{l}\text { Levene, Michelle; St George's University of London, } \\
\text { Enguita, Francisco; Universidade de Lisboa Associacao para a Investigacao } \\
\text { e Desenvolvimento da Faculdade de Medicina, Instituto de Medicina } \\
\text { Molecular } \\
\text { Bax, Bridget E.; St George's University of London }\end{array}$ \\
\hline \multicolumn{2}{|c|}{ microRNA, MNGIE, qPCR } \\
\hline
\end{tabular}

\section{SCHOLARONE \\ Manuscripts}




\section{Declaration}

I declare that the following manuscript has not been previously published nor submitted simultaneously for publication to any other journal. 


\section{Title}

Discovery profiling and bioinformatics analysis of serum microRNA in Mitochondrial NeuroGastrolntestinal Encephalomyopathy (MNGIE)

\section{Shortened title}

Discovery profiling of serum microRNAs in MNGIE

\section{Authors}

Levene, M. ${ }^{1}$, Enguita, F.J. ${ }^{2}$ and Bax, B.E. ${ }^{1}$

${ }^{1}$ St George's University of London, Molecular and Cell Sciences Institute, Cell Biology and Genetics Research Centre, United Kingdom.

${ }^{2}$ Instituto de Medicina Molecular, Faculdade de Medicina, Universidade de Lisboa, Av. Egas Moniz, 1649-028 Lisboa, Portugal. 
1

2

3

4

5

6

7

8

9

\begin{abstract}
Mitochondrial neurogastrointestinal encephalomyopathy (MNGIE) is a rare and fatal inherited metabolic disorder due to mutations in the nuclear TYMP gene and leads to a deficiency in the enzyme thymidine phosphorylase. This results in an accumulation of the deoxynucleosides, thymidine and deoxyuridine in the cellular and extracellular compartments, ultimately leading to mitochondrial failure. The understanding of the precise molecular mechanisms that underlie the disease pathology is limited, being hampered by the rarity of the disorder. Expression profiling of serum based mircoRNAs and subsequent bioinformatical analyses provide an approach to facilitate the identity of dysregulated genes and signalling pathways potentially involved in the pathogenesis of MNGIE.
\end{abstract}




\section{Introduction}

Mitochondrial Neurogastrointestinal Encephalomyopathy (MNGIE) is an ultra- rare inherited disease which manifests clinically as severe gastrointestinal dysmotility, sensorimotor peripheral neuropathy, severe muscle weakness and progressive leukoencephalopathy. The condition is relentlessly progressive with patients usually dying from a combination of nutritional failure and muscular disability at an average age of 37.6 years [1]. MNGIE results from a nuclear TYMP gene mutation causing a deficiency in thymidine phosphorylase (EC 2.4.2.4) [2] and leads to an accumulation of the deoxynucleosides, thymidine and deoxyuridine in tissues and body fluids. This results in elevated intracellular concentrations of their corresponding triphosphates and imbalances in the mitochondrial deoxyribonucleoside triphosphate pools, which in turn, interfere with normal mitochondrial DNA (mtDNA) replication, causing multiple deletions, somatic point mutations and depletion of mtDNA $[3,4]$. The synthesis of mtDNA in post-mitotic cells is dependent on the supply of deoxynucleosides via the mitochondrial nucleotide salvage pathway and thus tissues such as skeletal muscle and nervous system are most affected in MNGIE [3-5]. The precise mechanistic details of how mtDNA defects influence the disease phenotype remain unclear.

An area of genomics which is proving to be invaluable in facilitating the understanding of molecular pathways and gene regulatory networks in rare diseases is the study of small noncoding RNAs, and in particular, the genome-encoded microRNAs (miRNAs). MiRNAs are small single-stranded non-coding RNAs ranging from 19-25 nucleotides in size and are transcribed from endogenous nuclear transcripts. By base pairing to partially complementary sequences in mRNA, miRNAs down-regulate gene expression by inhibiting translation or directing degradation of mRNAs [6]. MiRNAs are expressed in all cell types 
and are secreted in to the extracellular environment, including the blood plasma and urine [7]. Individual microRNAs can target many different mRNAs, and individual mRNAs can contain multiple binding sites for different microRNAs, thus creating a complex regulatory network.

Expression profiling has enabled the identification of specific miRNAs which regulate a diverse range of biological processes including differentiation, proliferation, cellular development, metabolism, and apoptosis [8]. However, there is increasing evidence to suggest that dysregulated miRNA profiles are associated to many diseases such as cancer [9] and Parkinson's disease [10]. It has been suggested that the dysregulation of miRNA leads to a pathophysiological modulation of cells and therefore leading to the development or progression of many disorders [11]. Thus the analysis of mRNA and miRNA expression signatures in diseased tissues and extracellular fluids, and their comparison with molecular signatures of healthy individuals provides a powerful experimental approach for furthering the understanding of the molecular mechanisms of disease pathogenesis.

We report here a discovery phase investigation where the expression profiles of miRNA in the serum of MNGIE patients were analysed and compared to age/gender matched healthy controls. A bioinformatical approach of the data was applied to link signalling pathways with predicted miRNA gene targets with the aim of providing further understanding of the intracellular processes that are perturbed in MNGIE pathogenesis. 


\section{Methods}

Serum collection

This study was approved by the Royal Marsden Research Ethics Committee and informed consent was obtained from all participants prior to performing venesection. Venous blood was collected from five MNGIE patients and five healthy age/gender matched controls into silicon-coated vacutainers (Beckman Dickinson, UK) and gently mixed by inversion followed by standing upright for 30 minutes at room temperature to enable the blood to clot. The vacutainers were centrifuged at 1,300 x g (Beckman GPR centrifuge) for 10 minutes at room temperature, followed by aspiration the serum supernatant into RNase-free microtubes and stored at $-80^{\circ} \mathrm{C}$ until analysis

\section{RNA extraction}

RNA was extracted from the serum using Qiagen's miRNeasy serum and plasma kit with the addition of Exiqon's synthetic spike-ins (UniSp2/UniSp4/UniSp5) incorporated into the QIAzol lysis buffer, as an internal RNA extraction control.

\section{CDNA preparation}

Each serum RNA extraction was used as the template RNA for the preparation of cDNA. Briefly, $16 \mu \mathrm{L}$ microliters of RNA template were incorporated into a mastermix containing the following; $16 \mu \mathrm{L}$ Exiqon's Reverse Transcription (RT) reaction buffer (containing the RT primer), $36 \mu \mathrm{L}$ RNase-free water, $8 \mu \mathrm{L}$ enzyme mix (containing Poly(A) polymerase and Reverse Transcriptase) and $4 \mu \mathrm{L}$ synthetic spike-in (UniSp6). In a single reaction step, the mature miRNAs were polyadenylated and reverse transcribed into CDNA. The reaction mix 
was incubated for 60 minutes at $42^{\circ} \mathrm{C}$ followed by 5 minute incubation at $95^{\circ} \mathrm{C}$ and immediately cooled at $4^{\circ} \mathrm{C}$. The incorporation of the UniSp6 spike-in was used as an internal amplification control to evaluate the reverse transcription efficiency of each sample. Additionally, a no template control was used to assess for any RNA contamination and background filtration.

\section{qPCR using miRCURY LNA Universal RT microRNA PCR Human panel I+II}

Eighty microlitres of cDNA were combined with a reaction mix containing $3920 \mu \mathrm{L}$ of nuclease-free water and $4000 \mu \mathrm{L}$ of 2 x miCURY SYBR Green master mix. Each 384-well plate (see the Exiqon website for details of the miRNA primer hybridised to the panels) was briefly centrifuged prior to adding $10 \mu \mathrm{L}$ of the PCR master mix: CDNA mix to each well. The plate was sealed and briefly centrifuged to drive the contents to the bottom of the plate. The PCR was performed on Roche LightCycler ${ }^{\circledR} 480$ with the following cycling conditions; a PCR activation step for 10 minutes at $95^{\circ} \mathrm{C}$, followed by 45 amplification cycles set at $95^{\circ} \mathrm{C}$ for 10 seconds with the inclusion of a 1 minute ramp cooling (rate set at $1.6^{\circ} \mathrm{C} /$ second). A melt curve analysis was completed prior to exporting the data.

\section{qPCR data Quality Control}

On completion of the PCR programme, raw $\mathrm{Cq}$ values were exported and subjected to quality control checks including, melt curve analysis, detection of haemolysis, amplification efficiency and an evaluation of the negative control. Melt curve analysis enabled for the assessment of the specificity of each PCR reaction. Haemolysis was assessed by measuring the ratios of the raw $\mathrm{Cq}$ values of two specific miRNAs, miR-451 (highly abundant in erythrocytes) and miR-23a-3p (stably expressed in serum and not affected by haemolysis). A 
ratio with values $>7$ indicated the risk of haemolysis in the sample preparations and were removed from the dataset. The values of the synthetic spike-in (UniSp6) were used to determine the amplification efficiency.

The raw Cq values which passed the quality control criteria were subjected to normalisation prior to analysis to correct for overall differences between samples. Normalisation was based on the average of the assays which were detected in all the samples. A global mean was taken from the $\mathrm{Cq}$ values of all these miRNAs. Expression differences were calculated between the $\Delta \mathrm{Cq}$ values of the control and patient samples. The fold change was calculated as $2-(\Delta \Delta \mathrm{Cq})$, whereby values $>1$ were considered as upregulation and those values $<1$ were considered as downregulation.

\section{Bioinformatical analysis}

Differentially expressed miRNAs were assigned to a list of either up- or down-regulated expression (>2 fold, $p$ ? 0.05 Benjamini-Hochberg adjusted) and imported into the miRWalk database for miRNA interactions [12]. Candidate target genes that were common to four bioinformatic prediction algorithms (miRanda, PICTAR2, PITA and Targetscan) were identified. To assign biological function to the putative target genes of the miRNAs, a gene ontology (GO) enrichment analysis was conducted using the online Database for Annotation, Visualization and Integrated Discovery (DAVID) [13,14]. Cellular pathway enrichment analyses were conducted using the KEGG database; whereby only predicted genes with $p$ ? 0.01 and a false discovery rate ?0.01 were included. 
To unveil the relationships of the differentially expressed miRNAs, networks were generated between multiple miRNAs (at least 8 miRNA) and their potential gene targets, using the NAVIGATOR software [15]. 
Results

qPCR Data $Q C$

For the melt curve analysis, all reactions which had multiple melting points were removed from the dataset. The haemolysis assessment indicated that all of the samples were free from red blood cell contamination. Amplification efficiencies calculated to be $<1.6$ were removed from the data set.

miRNA differential expression

Compared to age and sex matched healthy controls, 50 miRNAs were significantly upregulated by 2.0 to 14.7 fold, $p$ ? 0.05 , Figure 1 , and whilst 32 were downregulated between -12.1 and -2.1 fold, $p$ ? 0.05 in the serum of patients with MNGIE.

The bioinformatical analyses focused on the upregulated miRNA where by the predicted gene targets were subjected to further enrichment analysis to determine possible relevance to MNGIE. Gene ontologies revealed regulation of transcription to be the highest ranked $\underline{\text { biological process, Synapses were the most enriched cellular component and transcriptional }}$ regulatory activity was the highest ranked for molecular function. Interestingly, the KEGG pathway analysis revealed the most enriched pathways to include axon guidance, endocytosis, and regulation of actin cytoskeleton. A literature search of these key findings can reveal a plethora of studies, some specific to MNGIE and others open for interpretation. 
Figure 1. Differentially expressed miRNAs in MNGIE patient serum compared to control. Differentially expressed miRNAs in serum of patients with MNGIE relative to healthy controls. Upregulated miRNAs are indicated by red bars (2.0 -14.7 fold, $p<0.05)$, and down-regulated in by blue bars (-12.1- 2.1 fold, $p<0.05)$.

Due to the large volume of data generatedfrom the bioinformatical analyses, this paper will only focus on the results obtained from the bioinformatics analyses of upregulated miRNAs. Using the miRWalk database, 3338 candidate target genes were identified that were common to the four bioinformatic prediction algorithms employed. These predicted gene targets were subjected to further enrichment analysis to determine possible relevance to MNGIE.

A literature search of these key findings can reveal a plethora of studies, some specific to MNGIE and others open to interpretation.

Gene ontologies and pathway enrichment

An analysis of gene ontology terms associated with the target genes revealed the following top enrichments: regulation of transcription and transcription for biological processes; synapse and neuron for cellular components; transcription regulator activity and transcription factor activity for molecular function (Table 1). A KEGG pathway enrichment analysis of the predicted genes ( $p$ ? 0.01; False Discovery Rate [0.01) revealed 15 different pathways, with the most enriched pathways being axon guidance, endocytosis, and regulation of actin cytoskeleton (Table 2). 
Table 1: Gene ontology terms for the upregulated miRNA predicted gene targets. Gene ontology enrichment of potential targets of up-regulated miRNAs for biological processes, cellular components and molecular function.

Table 2: KEGG pathways for the upregulated miRNA predicted gene targets. A KEGG pathway enrichment analysis of the predicted genes ( $\mathrm{p}$ ? 0.01; False Discovery Rate ?0.01).

miRNA - putative gene target network

By constructing a network of the putative relationships between miRNAs and candidate genes targeted by more than 8 upregulated miRNAs we were able to identify two targets of potential relevance to MNGIE, QKI and NFIB (Figure 2); these genes were the potential targets of 14 and 15 miRNAs, respectively, indicating that they are subjected to tight regulatory control.

Figure 2: NAVIGATOR network of multiple upregulated miRNA gene targets. MiRNA-target network for 31 of the up-regulated miRNAs in serum. Yellow ring represents genes targeted by 8 to 9 miRNAs, blue ring containing labelled genes targeted by 10 to 12 miRNAs. Centrally placed genes are targeted by 13 (PURB), 14 (QKI) and 15 (NFIB) miRNAs. 


\section{Discussion}

Studies have shown that aberrant miRNA profiles are implicated in many pathological states [16]. The discovery that abnormal expression profiles of miRNAs are implicated in muscular and neurological disorders $[17,18]$ led us to investigate their involvement in MNGIE. Therefore the aim of this study was to determine whether miRNA profiles were aberrantly expressed in the serum of MNGIE patients compared to health individuals and to apply a bioinformatical approach to establish the genetic implications of such differences.

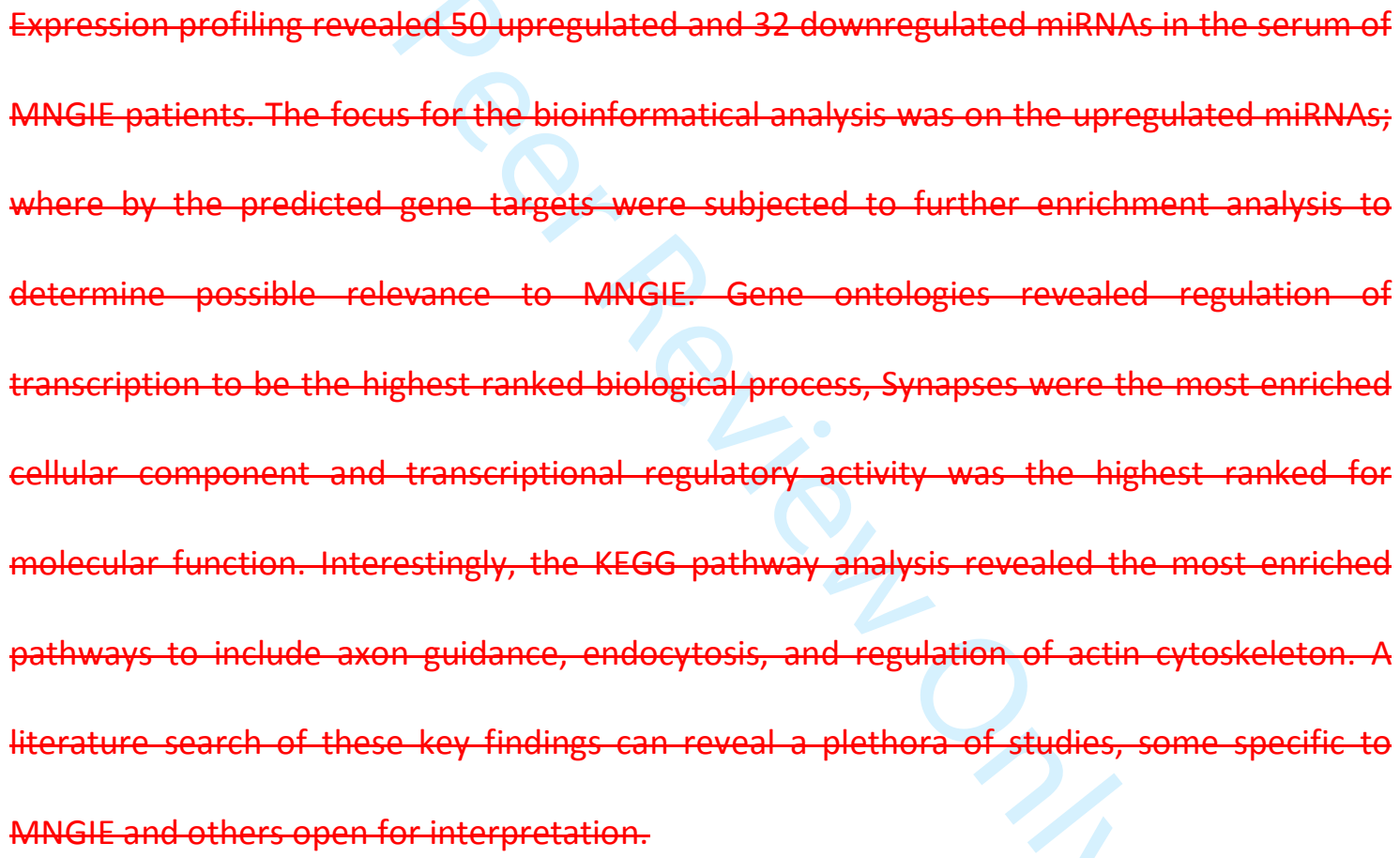

Ultrastructural of nerve biopsies of patients with MNGIE have revealed diffuse demyelinating polyneuropathy with gradual axonal loss, axonal neuropathy, and abnormal mitochondrial in axons and Schwann cells [19]. A study which focused on the optic neuropathy in MNGIE revealed hyper-myelinated axons and a temporal axonal loss in the optic nerve [20]. Our KEGG analysis revealed axonal guidance as one of the most enriched 
pathways and lends support to the involvement of the aberrantly expressed miRNAs in the regulation of genes involved in axonal processes.

Another enriched pathway was actin cytoskeleton; the regulation of actin cytoskeleton is essential for many biological processes such as immune surveillance, tissue repair and regeneration, angiogenesis and cell movement. Studies have shown that the dysregulation of cytoskeletal proteins contributes to various disease states such as cancer, neurological and musculoskeletal disorders [21]. It could be inferred that the neurological or skeletal muscle irregularities identified in MNGIE could relate to the dysregulation of the actin cytoskeleton.

It is prudent to acknowledge that this is an early discovery phase study and further experiment-investigation is required to ascertain a definite link between these molecular findings and the disease pathology of MNGIE. In particular, bioinformatical analysis is based on citations in the literature and therefore studies which dominate the field such as cancer or Parkinson's, can lead to a bias in the enrichment pathway analysis. This is particularly true for MNGIE, since the overexpression of where thymidine phosphorylase - (also known as platelet-derived endothelial cell growth factor or PD-ECGF) is positively correlated with angiogenesis and a poor prognosis in a number of cancers reported to be elevated in - a number of cancers. [22].

The analysis of the network relations between multiple miRNAs targeting single genes enabled a visualisation of which genes are subjected to a tighter regulatory control. Figure 2 illustrates two genes which are targeted by the highest number of miRNAs, the QKI (quaking) gene being targeted by 14 miRNA and NFIB (Nuclear Factor IB) gene targeted by 
15 miRNA. The QKI gene encodes for a family of RNA-binding proteins which are abundantly expressed in glial cells, including oligodendrocytes and astrocytes in the central nervous system and Schwann cells in the peripheral nervous system $[23,24]$. Studies have shown that QKI proteins play a key role in Schwann cell differentiation and myelination and the ensheathment of axons in the peripheral nervous system [25]. NFIB encodes for the Nuclear Factor I B, a site-specific transcription factor which is involved in the maintenance of neural progenitor cells, regulation of glial cell differentiation, neuronal migration and development of axons $[26,27]$.

Given that predicted gene targets of the upregulated miRNAs are strongly enriched in processes and functions related to axonal support and that demyelinating peripheral neuropathy and axonal degeneration represent some of the main clinical features of MNGIE, these miRNAs deserve further evaluation as predictive biomarkers of the neurological aspects of MNGIE. However this needs to be explored with some caution because circulating miRNAs have not been fully elucidated regarding their physiological role and their affects on gene expression in several types of tissues.

This was a small study, where only 5 age and sex matched patients were studied. The limited number of MNGIE samples available prevents mass-screening investigations for candidature miRNAs; therefore the use of qPCR panel arrays for this study enabled maximal screening from a minimal sample input. Serum was selected as the biofluid of choice for this type of investigation because it is easily obtained whilst being minimally invasive. Also the use of - Sserum instead of plasma was employed since plasma samples are invariably contaminated by platelets and significantly affect the levels of miRNA detected [28]. 
A second extension study is currently underway; whereby a further 10 patient and 10 control (age/gender matched) samples are being examined to determine if this additional cohort will confirm these initial findings reported here. Significant miRNAs from both the discovery phase and extension study will be subjected to intensive bioinformatical analysis and validated in an independent cohort of MNGIE patients.

The only other study which has investigated the involvement of miRNAs in MNGIE is that of Yong et al. [29]_2015)_who conducted a miRNA expression profile in whole blood of a single patient with MNGIE and a cohort of heterozygous family members [29]. However, no quality control checks were conducted to assess haemolysis, which has been shown to alter miRNA profiles [30]. The authors did not perform a downstream analysis of the differentially expressed miRNAs, but focused on examining the regulatory role of miRNAs over the TYMP gene; consequently the study did not identify a significant association with the differentially expressed miRNAs.

MicroRNA expression profiling and bioinformatics analysis is a powerful approach for elucidating the potential gene targets and target pathways in disease states; however it comes with many challenges. It is essential to implement precautionary measures to reduce technical variation, however excluding technical variance, miRNA species themselves possess a great deal of variability; being short in length, heterogeneous with their GC content [31] and mature miRNA sequences can differ by only one nucleotide, thus creating difficulties with purification [32]. Prior to downstream miRNA applications, the preanalytical variables need to be considered, such as individual variance from sample population including, smoking, fasting states and premedication. Therefore as a minimum, 
1

2

3

4

5

6

7

8

9

10

healthy controls need to be age and gender matched to the study group, whilst ensuring the sample preparation and storage conditions is consistent amongst all groups.

The current literature surrounding MNGIE is informative, enabling further comprehension of such a complex disorder and with the introduction of miRNA to this research field, this has broadened the capabilities to investigate MNGIE from a genomic perspective.

\section{Conclusion}

Profiling miRNA expression patterns can be informative in terms of biological relevance and further understanding of gene dysregulation in disease states [16]. This discovery phase has revealed that the serum miRNA expression profiles of patients with MNGIE are different to healthy controls. Bioinformatical analysis of these altered miRNA expressions translated to 3338 predicted target genes and a number of enriched pathways relevant to the MNGIE phenotype. Further evaluation of these target genes alongside the extension study will facilitate further understanding of the underlying pathogenic mechanisms of MNGIE.

Acknowledgments:

The authors gratefully acknowledge the support of the Purine Metabolic Patients' Association (PUMPA) charity for this research. 


\section{References}

[1] M. Hirano, Y. Nishigaki, and R. Martí. Mitochondrial neurogastrointestinal encephalomyopathy (MNGIE): a disease of two genomes, Neurologist. 2004, 10, 817.

[2] I. Nishino, A. Spinazzola, and M. Hirano. Thymidine phosphorylase gene mutations in MNGIE, a human mitochondrial disorder, Science. 1999, 283, 689-692.

[3] P. Ferraro, G. Pontarin, L. Crocco, S. Fabris, P. Reichard, and V. Bianchi. Mitochondrial deoxynucleotide pools in quiescent fibroblasts: a possible model for mitochondrial neurogastrointestinal encephalomyopathy (MNGIE), J. Biol. Chem. 2005, 280, 2447224480.

[4] E. González-Vioque, J. Torres-Torronteras, A. L. Andreu, and R. Martí. Limited dCTP availability accounts for mitochondrial DNA depletion in mitochondrial neurogastrointestinal encephalomyopathy (MNGIE), PLoS Genet. 2011, 7, e1002035e1002035.

[5] G. Pontarin, P. Ferraro, M. L. Valentino, M. Hirano, P. Reichard, and V. Bianchi. Mitochondrial DNA depletion and Thymidine Phosphate Pool Dynamics in a Cellular Model of Mitochondrial Neurogastrointestinal Encephalomyopathy, J. Biol. Chem. 2006, 281, 22720-22728.

[6] D. P. Bartel. MicroRNAs: genomics, biogenesis, mechanism, and function, Cell. 2004, 116, 281-297.

[7] J. A. Weber et al. The microRNA spectrum in 12 body fluids, Clin. Chem. 2010, 56, 1733-1741.

[8] J. Wang, J. Chen, and S. Sen. MicroRNA as Biomarkers and Diagnostics, J. Cell. Physiol. 2016, 231, 25-30.

[9] J. Lu et al. MicroRNA expression profiles classify human cancers, Nature. 2005, 435, 834-838.

[10] R. Margis, R. Margis, and C. R. M. Rieder. Identification of blood microRNAs associated to Parkinson's disease, J. Biotechnol. 2011, 152, 96-101.

[11] G. Hu, K. M. Drescher, and X.-M. Chen. Exosomal miRNAs: Biological Properties and Therapeutic Potential, Front. Genet. 2012, 3, 56.

[12] H. Dweep, N. Gretz, and C. Sticht. miRWalk database for miRNA-target interactions, Methods Mol. Biol. 2014, 1182, 289-305.

[13] D. W. Huang, B. T. Sherman, and R. A. Lempicki. Systematic and integrative analysis of large gene lists using DAVID bioinformatics resources, Nat. Protoc. 2009, 4, 44-57.

[14] D. W. Huang, B. T. Sherman, and R. A. Lempicki. Bioinformatics enrichment tools: paths toward the comprehensive functional analysis of large gene lists, Nucleic Acids Res. 2009, 37, 1-13.

[15] K. R. Brown et al. NAViGaTOR: Network Analysis, Visualization and Graphing Toronto, Bioinformatics. 2009, 25, 3327-9. 
[16] B. C. Bernardo, F. J. Charchar, R. C. Y. Lin, and J. R. McMullen. A microRNA guide for clinicians and basic scientists: background and experimental techniques, Heart. Lung Circ. 2012, 21, 131-142.

[17] I. Eisenberg et al. Distinctive patterns of microRNA expression in primary muscular disorders, Proc. Natl. Acad. Sci. U. S. A. 2007,104, 17016-17021.

[18] D. A. Galloway and C. S. Moore. miRNAs As Emerging Regulators of Oligodendrocyte Development and Differentiation, Front. cell Dev. Biol. 2016, 4, 59.

[19] G. Said et al. Clinicopathological aspects of the neuropathy of neurogastrointestinal encephalomyopathy (MNGIE) in four patients including two with a Charcot-MarieTooth presentation, J. Neurol. 2005, 252, 655-662.

[20] F. Ross-Cisneros et al. Optic Neuropathy in a Case of Mitochondrial Neurogastrointestinal Encephalomyopathy (MNGIE): Ultrastructural Features and Mitochondrial DNA analysis, iovs. 2011, 52, 3872.

[21] S. H. Lee and R. Dominguez. Regulation of actin cytoskeleton dynamics in cells, Mol. Cells. 2010, 29, 311-25.

[22] Y. Y. Elamin, S. Rafee, N. Osman, K. J. O Byrne, and K. Gately. Thymidine Phosphorylase in Cancer; Enemy or Friend?, Cancer Microenviron. 2016, 9, 33-43.

[23] R. J. Hardy et al. Neural cell type-specific expression of QKI proteins is altered in quakingviable mutant mice, J. Neurosci. 1996, 16, 7941-9.

[24] R. J. Hardy. QKI expression is regulated during neuron-glial cell fate decisions, $J$. Neurosci. Res. 1998, 54, 46-57.

[25] D. Larocque et al. The QKI-6 and QKI-7 RNA Binding Proteins Block Proliferation and Promote Schwann Cell Myelination, PLoS One. 2009, 4, e5867.

[26] B. Cebolla and M. Vallejo. Nuclear factor-I regulates glial fibrillary acidic protein gene expression in astrocytes differentiated from cortical precursor cells, J. Neurochem. 2006, 97, 1057-70.

[27] B. Deneen, R. Ho, A. Lukaszewicz, C. J. Hochstim, R. M. Gronostajski, and D. J. Anderson. The transcription factor NFIA controls the onset of gliogenesis in the developing spinal cord, Neuron. 2006, 52, 953-68.

[28] A.J.Mitchell et al. Platelets confound the measurement of extracelluar miRNA in archived plasma, Sci. Rep. 2016, 6, 32651.

W. Liand H. Yue. Thymidine phosphorylase: A potential new target for treating eardiovascular disease, Trends Cardiovasc. Med. 2017.

[29] F. L. Yong, C. W. Wang, and K. S. Tan. MicroRNA expression profile of a Malaysian Bajau family with familial mitochondrial neurogastrointestinal encephalomyopathy, Genet. Mol. Res. 2015, 14, 13172-13183.

[30] T. Blondal et al. Assessing sample and miRNA profile quality in serum and plasma or other biofluids, Methods. 2013, 59, S1-S6.

[31] C. C. Pritchard, H. H. Cheng, and M. Tewari. MicroRNA profiling: approaches and considerations, Nat. Rev. Genet. 2012, 13, 358-369. 
[32] V. Benes and M. Castoldi. Expression profiling of microRNA using real-time quantitative PCR, how to use it and what is available, Methods. 2010, 50, 244-249. 


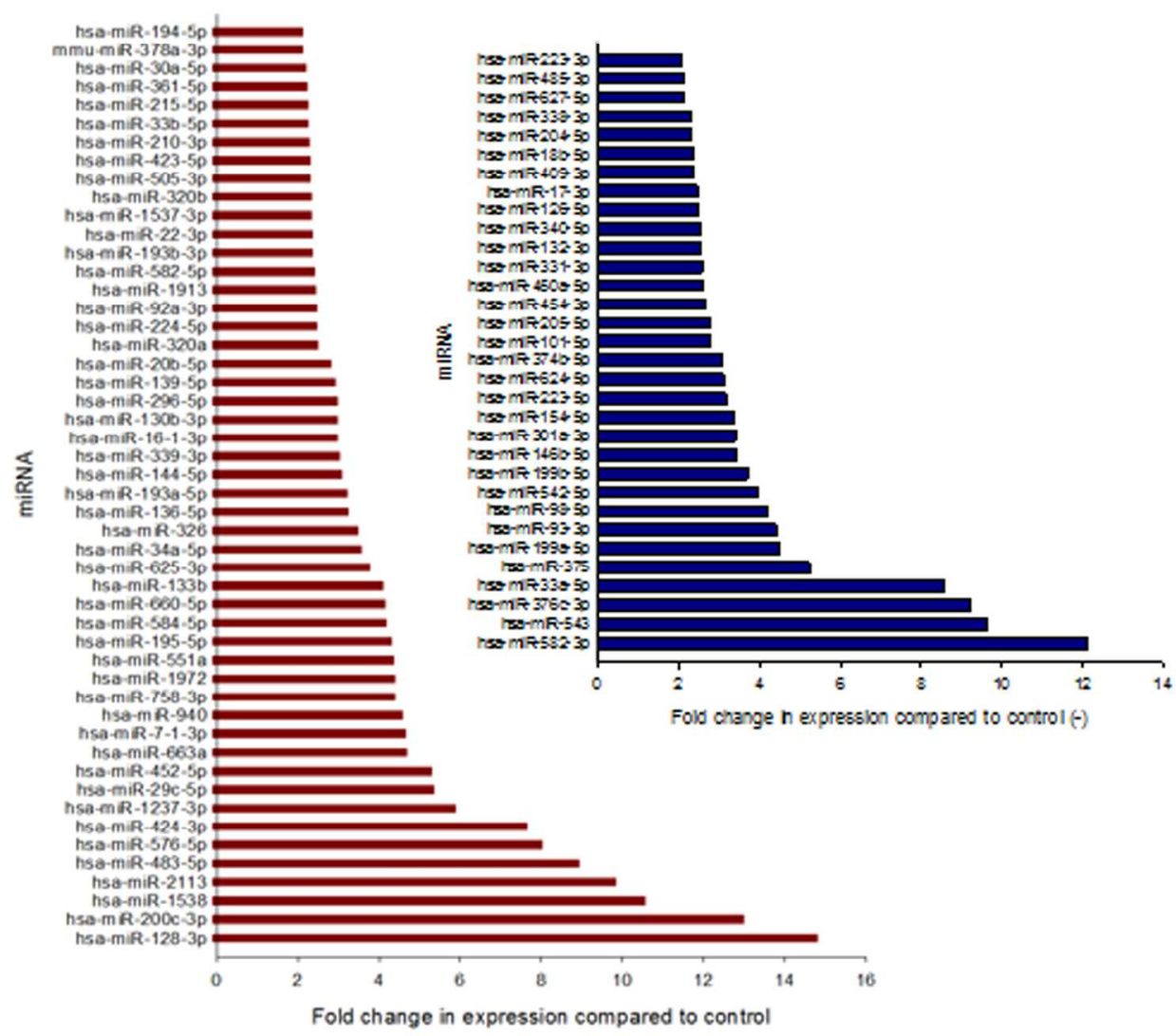

Differentially expressed miRNAs in MNGIE patient serum compared to control.

$137 \times 130 \mathrm{~mm}(300 \times 300$ DPI $)$ 


\begin{tabular}{|l|l|l|l|}
\hline Term & Count & $p$ value & FDR \\
\hline Biological Processes & 698 & $1.03 \mathrm{E}-34$ & $1.94 \mathrm{E}-31$ \\
\hline Regulation of transcription & 575 & $3.70 \mathrm{E}-30$ & $6.95 \mathrm{E}-27$ \\
\hline Transcription & \multicolumn{4}{|l|}{} & $8.40 \mathrm{E}-25$ \\
\hline $\begin{array}{l}\text { Regulation of transcription from RNA polymerase II } \\
\text { promoter }\end{array}$ & 253 & $4.46 \mathrm{E}-28$ & $3.74 \mathrm{E}-19$ \\
\hline Regulation of RNA metabolic process & 484 & $1.99 \mathrm{E}-22$ & $4.70 \mathrm{E}-18$ \\
\hline Positive regulation of transcription & 195 & $2.50 \mathrm{E}-21$ & $3.33 \mathrm{E}-15$ \\
\hline Cellular Component & 122 & $2.42 \mathrm{E}-16$ & $8.33 \mathrm{E}-14$ \\
\hline Synapse & 118 & $5.31 \mathrm{E}-16$ & $9.32 \mathrm{E}-11$ \\
\hline Neuron projection & 142 & $6.23 \mathrm{E}-12$ & $1.05 \mathrm{E}-10$ \\
\hline Extrinsic to membrane & 221 & $7.02 \mathrm{E}-12$ & $2.08 \mathrm{E}-10$ \\
\hline Golgi apparatus & 184 & $1.39 \mathrm{E}-11$ & $4.91 \mathrm{E}-09$ \\
\hline Cell projection & 466 & $3.28 \mathrm{E}-10$ & $1.44 \mathrm{E}-08$ \\
\hline Plasma membrane part & 140 & $9.62 \mathrm{E}-10$ & $1.65 \mathrm{E}-08$ \\
\hline Cell junction & 194 & $1.10 \mathrm{E}-09$ & $2.36 \mathrm{E}-08$ \\
\hline Endomembrane system & 147 & $1.58 \mathrm{E}-09$ & $1.03 \mathrm{E}-07$ \\
\hline Nucleoplasm part & 57 & $6.85 \mathrm{E}-09$ & $1.21 \mathrm{E}-24$ \\
\hline Axon & 435 & $7.30 \mathrm{E}-26$ & $1.44 \mathrm{E}-19$ \\
\hline Molecular Function & 295 & $8.72 \mathrm{E}-21$ & $1.19 \mathrm{E}-13$ \\
\hline Transcription regulator activity & 190 & $7.23 \mathrm{E}-15$ & $9.22 \mathrm{E}-12$ \\
\hline Transcription factor activity & 555 & $5.57 \mathrm{E}-13$ & \\
\hline Sequence-specific DNA binding & \multicolumn{5}{|l}{} \\
\hline DNA binding &
\end{tabular}

Gene ontology terms for the upregulated miRNA predicted gene targets

$137 \times 95 \mathrm{~mm}(300 \times 300 \mathrm{DPI})$ 


\begin{tabular}{|l|l|l|l|}
\hline Term & Count & P Value & FDR \\
\hline Axon guidance & 54 & $2.70 \mathrm{E}-11$ & $3.32 \mathrm{E}-10$ \\
\hline Endocytosis & 65 & $1.08 \mathrm{E}-09$ & $1.33 \mathrm{E}-08$ \\
\hline Regulation of actin cytoskeleton & 65 & $8.77 \mathrm{E}-07$ & $1.08 \mathrm{E}-05$ \\
\hline Fc gamma R-mediated phagocytosis & 36 & $1.57 \mathrm{E}-06$ & $1.92 \mathrm{E}-05$ \\
\hline Wnt signalling pathway & 49 & $2.87 \mathrm{E}-06$ & $3.52 \mathrm{E}-05$ \\
\hline Melanogenesis & 36 & $4.69 \mathrm{E}-06$ & $5.76 \mathrm{E}-05$ \\
\hline Pathways in cancer & 84 & $2.90 \mathrm{E}-05$ & $3.56 \mathrm{E}-04$ \\
\hline TGF-beta signalling pathway & 31 & $3.88 \mathrm{E}-05$ & $4.76 \mathrm{E}-04$ \\
\hline Dilated cardiomyopathy & 32 & $4.82 \mathrm{E}-05$ & $5.91 \mathrm{E}-04$ \\
\hline Colorectal cancer & 30 & $5.08 \mathrm{E}-05$ & $6.23 \mathrm{E}-04$ \\
\hline Focal adhesion & 56 & $7.54 \mathrm{E}-05$ & $9.25 \mathrm{E}-04$ \\
\hline Renal cell carcinoma & 25 & $2.52 \mathrm{E}-04$ & $3.08 \mathrm{E}-03$ \\
\hline Circadian rhythm & 9 & $3.48 \mathrm{E}-04$ & $4.26 \mathrm{E}-03$ \\
\hline MAPK signalling pathway & 67 & $4.02 \mathrm{E}-04$ & $4.93 \mathrm{E}-03$ \\
\hline Chronic myeloid leukaemia & 25 & $7.96 \mathrm{E}-04$ & $9.73 \mathrm{E}-03$ \\
\hline
\end{tabular}

KEGG pathways for the upregulated miRNA predicted gene targets $136 \times 64 \mathrm{~mm}(300 \times 300 \mathrm{DPI})$ 


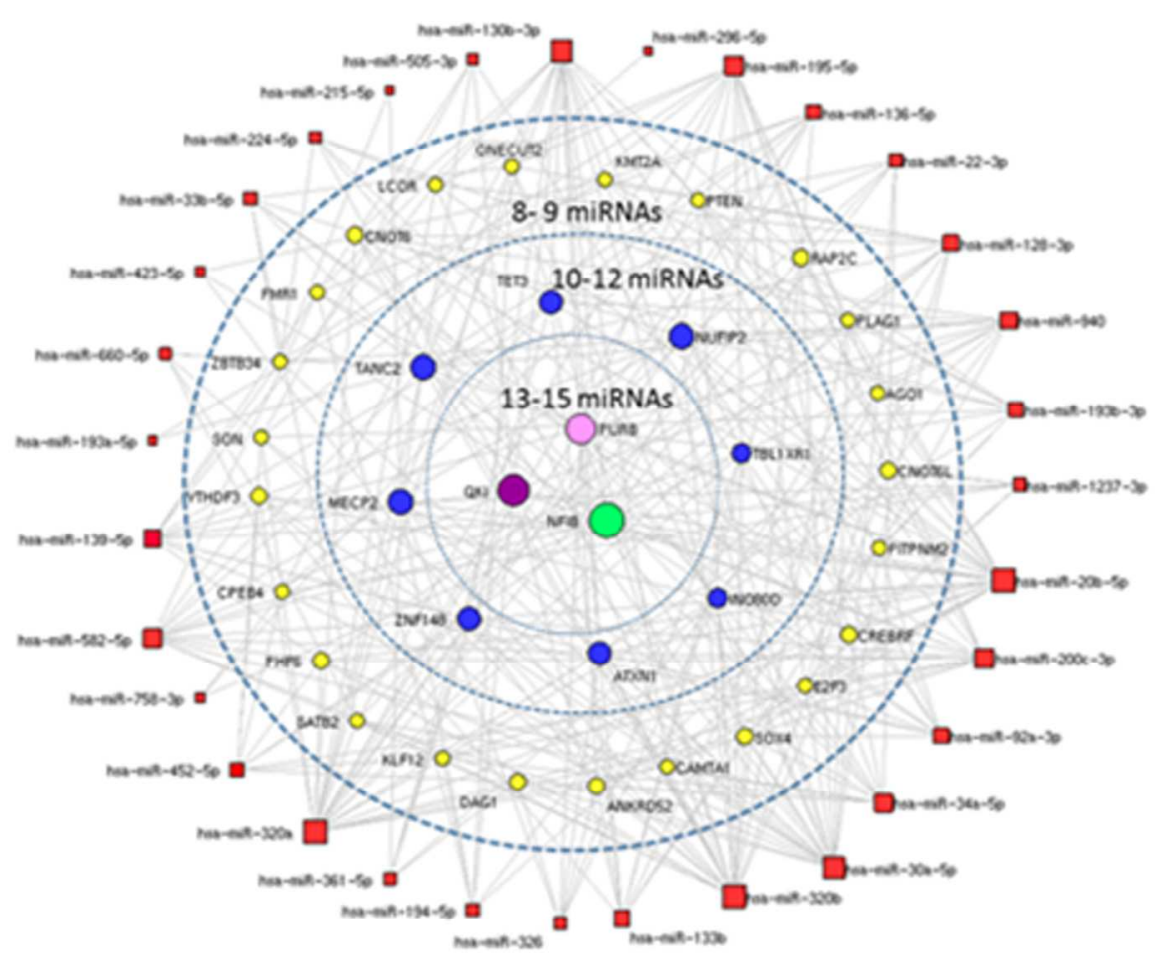

NAVIGATOR network of multiple upregulated miRNA gene targets $134 \times 119 \mathrm{~mm}(300 \times 300 \mathrm{DPI})$ 